\title{
Iterative LMI Approach for Robust Fault Detection Observer Design *
}

\author{
Haibo Wang ${ }^{\dagger} \quad$ Jianliang Wang ${ }^{\ddagger} \quad$ Jian Liu $\$ \quad$ James Lam ${ }^{\uparrow}$ \\ † School of Electrical \& Electronic Engineering, Nanyang Technological University, Singapore. ehbwang@ntu.edu.sg \\ ‡ School of Electrical \& Electronic Engineering, Nanyang Technological University, Singapore. ejlwang@ntu.edu.sg \\ $\S$ Honeywell Avionics Inc., Singapore. liujian@pmail.ntu.edu.sg \\ T Department of Mechanical Engineering, University of Hong Kong, Pokfulam Road, Hong Kong. jlam@hku.hk
}

\begin{abstract}
This paper deals with the robust fault detection (RFD) problem with the aid of the $H_{\infty}$ norm and $H_{-}$index optimization technique and the LMI approach. A necessary and sufficient condition is proposed for the design of RFD observers. RFD problem with structured uncertainties in the system matrices is also considered. Iterative LMI algorithms are proposed to design RFD observers to enhance the fault detection and to attenuate the effects due to unknown inputs and uncertainties. We also consider the RFD problem over finite frequency range in which case no full column rank constraint is required on $D$ for a system $(A, B, C, D)$. Numerical example is employed to demonstrate the effectiveness of the proposed methods.
\end{abstract}

\section{Introduction}

The research and application of model-based fault detection (FD) in automated processes have received considerable attention over the decades $[3,4,6,8,12,13,17]$ - A fault is usually defined as an unexpected change in a system, such as component malfunction and variations in operating condition, which tends to degrade the overall system performance. The purpose of FD is thus to determine the occurrence of fault in the system.

Robustness is the most fundamental problem in modelbased FD. In recent years, RFD observer design has been the subject of many published works. The $H_{\infty}$ norm optimization is a robust design method with the original motivation rooted in the consideration of various uncertainties. This technique is very suitable for handling uncertainities. After decades of development, it is now playing a leading role in treating robustness problems in control systems. It is therefore reasonable to use this technique in designing RFD systems. A number of approaches using $H_{\infty}$ norm optimization techniques have been developed for the design of RFD observers

\footnotetext{
* The work was supported in part by DSO National laboratories under grant DSOCL-01144, and by HKU CRCG Grant $10204304 / 19870$.
}

$[1,2,5,7,16,19]$.

Similar to the $H_{\propto}$ norm optimization technique, the $H_{-}$method has gained much attention recently, which aims to study the worst-case fault sensitivity performance of a fault detection observer. An $H_{-}$norm was defined in $[7,14]$ as the minimum nonzero singular value of the transfer function matrix from the fault to the residual output at the specific frequency of $\omega=0$. In $[1,15]$, this definition was further extended from the single frequency $\omega=0$ to a number of finite frequency ranges. However, due to the lack of efficient method to evaluate the proposed sensitivity measures, only numerical optimizations such as nonlinear programming, frequency grid method, and also genetic algorithm were used in evaluating these proposed sensitivity measures. It should also be pointed out that these sensitivity measures are not truly worst-case measures due to the exclusion of possible zero singular values of the transfer function matrix. A true worst-case fault sensitivity measure, $H_{-}$index, was proposed in $[10,11]$ to include the possible zero singular values of the transfer function matrix. Specifically, the $H_{-}$index was defined as the minimum singular value of the transfer function matrix over a given frequency range. Note that the constraint of "non-zero singular value" is absent here, which makes the $H_{-}$index no longer a norm (hence the term index). The frequency range can be either infinite (i.e. the entire frequency spectrum) or finite frequency intervals. Moreover, for infinite frequency spectrum case, a necessary. and sufficient condition in terms of LMIs was established for the proposed $H_{-}$index. The case of finite frequency range was handled by frequency weighting [10].

However, these papers considered only unknown inputs. System uncertainties were not considered. Strictly speaking, they can hardly be called RFD as system uncertainties were not considered. Therefore, a more general $H_{-}$method to solve RFD problem with system uncertainties is needed. This has not been systematically treated in previous fault detection studies $[1,7,10,11,14,15]$.

In this paper, we consider the robust fault detection problem for uncertain linear systems with the aid 
of the $H_{\infty}$ norm and $H_{-}$index optimization techniques and the LMI approach. First, a necessary and suffcient condition is proposed for the design of RFD observers in the absence of system uncertainties. Second, the RFD problem under structured uncertainties in the system matrices is also considered. Correspondingly, an iterative LMI algorithm is proposed to design RFD observers. The RFD problem over finite frequency range is also considered, in which case the condition on the rank of $D$ for a system $(A, B, C, D)$ is no longer required. Numerical simulations are used to illustrate the effectiveness of the results.

The paper is organized as follows. In Section 2, the RFD problem considered in this paper is introduced, together the performance index for facilitating the design of RFD observers. The problem is formulated into an LMI-based optimization and iterative algorithm to design RFD observers are given in Section 3. The RFD problem over finite frequency range is considered in Section 4. Section 5 provides numerical example and simulation results to illustrate the proposed approaches. Finally, some concluding remarks are provided in Section 6.

Throughout the paper, for a real symmetric $M$, we used $M>0(<0)$ to denote its positive (negative) definiteness. The spectral norm of $M$ is denoted by $\|M\|$. $\mathbb{Z}^{+}$is used to denote the set of all positive integers and " $*$ " denotes the symmetric entries of a symmetric matrix. All matrices, if their dimensions are not explicitly stated, are assumed to be compatible. For a vector function $h(t)$, we define its $L_{2}$ norm as

$$
\|h(t)\|_{2}=\sqrt{\int_{0}^{\infty} h^{T}(t) h(t) d t}
$$

\section{Problem Formulation}

Consider the following uncertain linear system

$$
\begin{aligned}
\dot{x}(t)= & (A+\Delta A(t)) x(t)+(B+\Delta B(t)) u(t) \\
& +D w(t)+F f(t) \\
y(t)= & (C+\Delta C(t)) x(t)+D_{w} w(t)+F_{f} f(t)
\end{aligned}
$$

where $x(t) \in \mathbb{R}^{n}$ is the state vector, $w(t) \in \mathbb{R}^{m}$ is the unknown input vector including uncertain disturbance, and process and measurement noises, $y(t) \in \mathbb{R}^{r}$ is the measurement vector, and $f(t) \in \mathbb{R}^{p}$ is the fault vector. Here, $A, B, D, F, C, D_{w}$ and $F_{f}$ are known constant matrices with appropriate dimensions. Furthermore, we assume that the pair $(C, A)$ is observable, the uncertain system $(1)$ is asymptotically stable, $w(t)$ is $L_{2}$-norm bounded, $f(t)$ is norm bounded, i.e. $f^{T}(t) f(t)$ is bounded. by

Suppose the uncertain structure of system $(1)$ is given

$$
\left[\begin{array}{cc}
\Delta A(t) & \Delta B(t) \\
\Delta C(t) & \diamond
\end{array}\right]=\left[\begin{array}{l}
F_{1} \\
F_{2}
\end{array}\right] E(t)\left[\begin{array}{ll}
H_{1} & H_{2}
\end{array}\right]
$$

where " $\diamond$ " denotes "do not care", $F_{1}, F_{2}, H_{1}$ and $H_{2}$ are known constant matrices with appropriate dimensions and $E(t)$ is an unknown matrix function of uncertain parameters satisfying

$$
E^{T}(t) E(t) \leq I
$$

The state observer under consideration is of the form

$$
\begin{aligned}
\ddot{\bar{x}}(t) & =\bar{A} \bar{x}(t)+B u(t)+L y(t) \\
\bar{y}(t) & =C \bar{x}(t)
\end{aligned}
$$

where $\bar{A}=A-L C$ and $L$ is the observer gain matrix to be designed. Define the error state and residual vector $r(t)$ as

$$
\begin{aligned}
& e(t)=x(t)-\bar{x}(t) \\
& r(t)=y(t)-\bar{y}(t)
\end{aligned}
$$

then it follows from (1) and (4) that the error and residual dynamic system can be described as

$$
\begin{aligned}
\dot{e}(t)= & \bar{A} e(t)+\left(F-L F_{f}\right) f(t) \\
& +\left[\begin{array}{lll}
\Delta A(t)-L \Delta C(t) & \Delta B(t) & D-L D_{w}
\end{array}\right] \tilde{w}(t) \\
r(t)= & C e(t)+\left[\begin{array}{lll}
\Delta C(t) & 0 & D_{w}
\end{array}\right] \tilde{w}(t)+F_{f} f(t)
\end{aligned}
$$

where

$$
\tilde{w}(t):=\left[\begin{array}{c}
x(t) \\
u(t) \\
w(t)
\end{array}\right]
$$

$\tilde{w}(t)$ can be considered as the new disturbance input vector.

For effective fault detection, the effect on sensitivity due to disturbance in the residual vector should be small while that due to faults should be large. Obviously, the $H_{\infty}$ norm optimization techniques can be used to handle disturbance attenuation problems. In the following, we introduce a notion to measure the effect due to faults.

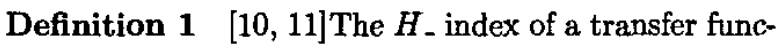
tion $G(s)$ over the frequency range $[0, \bar{\omega}]$ is defined as

$$
\|G(s)\|_{-}^{[0, \bar{\omega}]}=\inf _{\omega \in[0, \bar{\omega}]} \underline{\sigma}[G(j \omega)]
$$

where $\underline{\sigma}$ denotes the minimum singular value.

Remark 1 To indicate the dependency on the frequency

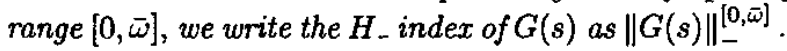
However, when the frequency range is clear from the context, we simply write $\|G(s)\|_{-}$.

Thus, the $H_{-}$index of the fault transfer function $H_{r f}(s)$ can be used to describe a measurement of the worst case fault sensitivity, where the fault transfer function $H_{r f}(s)$ from $f(t)$ to $r(t)$ are obtained as

$$
H_{r f}(s)=C(s I-\bar{A})^{-1}\left(F-L F_{f}\right)+F_{f}
$$

Based on the above motivation, we study the design problem of a robust fault detection observer for system (1) as follows:

Problem 1: For system (1) with the observer (4), determine an observer gain matrix $L$ such that

$\mathrm{P}$ (i) The error system (5) is asymptotically stable. 
$\mathrm{P}$ (ii) The fault detection 'noise-signal' gain ratio

$$
J=\frac{\gamma_{1}}{\gamma_{2}}
$$

is small where $\gamma_{1}>0, \gamma_{2}>0$, and

$$
\begin{aligned}
& \|r(t)\|_{2}<\gamma_{1}\|\check{w}(t)\|_{2} \\
& \left\|H_{r f}(s)\right\|_{-}>\gamma_{2}
\end{aligned}
$$

\section{Main Results}

For condition (8), we have the following.lemma which is a variant of Bound Real Lemma [20].

Lemma 1 [16]Consider the system (5) without uncertainties, given by

$$
\begin{aligned}
& \dot{e}(t)=\bar{A} e(t)+\left(D-L D_{w}\right) w(t)+\left(F-L F_{f}\right) f(t) \\
& r(t)=C e(t)+D_{w} w(t)+F_{f} f(t)
\end{aligned}
$$

Then the system (10) is asymptotically stable and satisfies (8) if and only if one of the following two equivalent conditions holds:

1. There exist $P>0$ and $L$ such that the following matrix inequality (MI) holds.

$$
\left[\begin{array}{cc}
A^{T} P+P A+C^{T} C & P\left(D-L D_{w}\right)+C^{T} D_{w} \\
-C^{T} L^{T} P-P L C & P\left(D_{w}\right) \\
\left(D-L D_{w}\right)^{T} P+D_{w}^{T} C & -\gamma_{1}^{2} I+D_{w}^{T} D_{w}
\end{array}\right]<0
$$

2. There exist $P>0$ and $\tilde{P}$ such that the following $L M I$ holds.

$$
\left[\begin{array}{cc}
A^{T} P+P A+C^{T} C & P D-\tilde{P} D_{w}+C^{T} D_{w} \\
-C^{T} \tilde{P}^{T}-\tilde{P} C & -\gamma_{1}^{2} I+D_{w}^{T} D_{w}
\end{array}\right]<0
$$

In this case, an observer gain is given by $L=P^{-1} \tilde{P}$.

On the other hand, for condition (9), the following lemma is introduced.

Lemma 2 [11]Assume that the system (10) is asymptotically stable, then inequality (9) holds if and only if there exist symmetric matrix $Q$ and matrix $L$ such that

$$
\left[\begin{array}{cc}
A^{T} Q+Q A+C^{T} C & Q\left(F-L F_{f}\right)+C^{T} F_{f} \\
-C^{T} L^{T} Q-Q L C & Q\left(-F_{f}\right. \\
\left(F-L F_{f}\right)^{T} Q+F_{f}^{T} C & -\gamma_{2}^{2} I+F_{f}^{T} F_{f}
\end{array}\right]>0
$$

From Lemmas 1 and 2, it is obvious that the following result holds.

Theorem 1 Consider the system (10) and suppose that $F_{f}$ is of full column rank. For given $\gamma_{1}>0$ and $\gamma_{2}>0$, the system (10) is asymptotically stable and satisfies (8) and (9) if and only if there exist matrix $P>0$, symmetric matrix $Q$ and matrix $L$ such that MIs (11) and (12) hold.
Now consider the system (5) which is suffering from uncertainties in the system matrices. At first, we introduce a useful lemma as follows.

Lemma $3 \quad[9,18]$ Let $A, F, H$ and $E(t)$ be real matrices of appropriate dimensions with $E(t)$ satisfying

$$
E^{T}(t) E(t) \leq I
$$

Then for any $\varepsilon>0$

$$
F E(t) H+(F E(t) H)^{T} \leq \varepsilon^{-1} F F^{T}+\varepsilon H^{T} H
$$

Thus, based on Lemma 3 and Theorem 1, we obtain the following theorem with proof omitted.

Theorem 2 Consider the system (5) and suppose that $F_{f}$ is of full column rank. For given $\gamma_{1}>0$ and $\gamma_{2}>0$, the system (5) is asymptotically stable and satisfies (8) and (9) if there exist matrix $P>0$, symmetric matrix $Q$, matrix $L$, and scalars $\varepsilon_{i}>0, i=1, \ldots, 3$, such that MIs (12) and (13) hold.

$$
\left[\begin{array}{ccccc}
W_{1} & 0 & 0 & P F_{1} & P\left(F_{1}-L F_{2}\right) \\
* & W_{2} & 0 & 0 & +C^{T} F_{2} \\
* & * & W_{3} & 0 & 0 \\
* & * & * & -\varepsilon_{2} I & 0 \\
* & * & * & * & -\varepsilon_{3} I \\
* & * & * & * & * \\
& P\left(D-L D_{w}\right)+C^{T} D_{w} & \\
\multicolumn{4}{c}{0} \\
0 \\
\multicolumn{4}{c}{0} \\
-\gamma_{1}^{2} I+D_{w}^{T} D_{w}+\varepsilon_{1} D_{w}^{T} F_{2} F_{2}^{T} D_{w}
\end{array}\right]<0
$$

$$
\begin{gathered}
\text { where } \\
W_{1}=A^{T} P+P A+C^{T} C-C^{T} L^{T} P-P L C \\
W_{2}=\left[\begin{array}{cc}
-\gamma_{1}^{2} I+\left\|F_{2}\right\| H_{1}^{T} H_{1}+\varepsilon_{3} H_{1}^{T} H_{1}^{T} & H_{1}^{T} \\
H_{1} & -\varepsilon_{1} I
\end{array}\right] \\
W_{3}=-\gamma_{1}^{2} I+\varepsilon_{2} H_{2}^{T} H_{2}
\end{gathered}
$$

Based on Theorem 2, it is possible to construct an iterative LMI algorithm to obtain a robust fault detection observer as given in the following schematic form.

Algorithm 1: Given system matrices $A, B, C, D, F$, $D_{w}, F_{f}, H_{1}, H_{2}, F_{1}$ and $F_{2}$. Let $\mu_{1} \geq 0$ and $\mu_{2} \geq 0$ be sufficiently small adjustable parameters. Set $i=0, j=$ 0 and $m \in \mathbb{Z}^{+}$to control the number of computational loops.

Step 1 Choose a sufficiently large $\gamma_{1}=\zeta$ and solve (13) to find feasible solutions $P$ and $\tilde{P}$ where $\tilde{P}=P L$. Compute $L=P^{-1} \tilde{P}$ and let $\gamma_{1}=\zeta$ and $\gamma_{2}=0$.

Step 2 (Main Iterative Steps)

(a) Substitute $L$ into (12) and (13) and find a feasible solution set of variables $P, Q$.

(b) Put $i=i+1$. With $P, Q$ obtained in Step 2(a) and with

$\gamma_{1}:=\gamma_{1}-\mu_{1}>\left\|D_{w}\right\| \quad, \quad \gamma_{2}:=\gamma_{2}+\mu_{2}<\left\|F_{f}\right\|$ 
find a feasible solution $L$ for LMIs (12) and (13). Store $L_{i}=L$ and $\frac{\gamma_{1}}{\gamma_{2}}$. Repeat Step 2(b). If a feasible solution cannot be found, then $L_{i}=L_{i-1}$.

(c) If the performance $\frac{\gamma_{1}}{\gamma_{2}}$ is less than some desired level, then a desired observer gain $L=L_{i}$ is found. STOP.

Step 3 Set $j=j+1$. If $j<m$, repeat Step 2, else STOP (the feasible solution cannot be found).

Remark 2 Step 1 is always feasible for sufficiently large $\gamma_{1}$ since, similar to item 2 of Lemma $1, M I$ (13) can be expressed in terms of $L M I$ by setting $\tilde{P}=P L$. Moreover, for given $P$ and $Q, M I s$ (12) and (13) become LMIs and a feasible solution $L$ can always be obtained provided that $\mu_{1}$ and $\mu_{2}$ are sufficiently small. Therefore, Step 2 can always provide a local improvement through each iteration.

Note that inequalities (12) and (13) in Theorem 2 are MIs in $P, Q$ and $L$. In the following an iterative LMI approach will be proposed based on Theorem 2 with proof omitted.

Theorem 3 Consider the system (5) and suppose that $F_{f}$ is of full column rank. For given $\gamma_{1}>0$ and $\gamma_{2}>0$, the system (5) is asymptotically stable and satisfies (8) and (9) if there exist matrix $P>0$, symmetric matrix $Q$, matrix $L$, and scalars $\varepsilon_{i}>0, i=1, \ldots, 3$, such that the following LMIs (14) and (15) hold for given matrices $P_{0}, Q_{0}, L_{0}$.

$\left[\begin{array}{cccc}M & Q F+C^{T} F_{f} & Q+C^{T} L^{T} & Q \\ * & N & 0 & F_{f}^{T} L^{T} \\ * & * & I & 0 \\ * & * & * & I\end{array}\right]>0$

$$
\left.\begin{array}{cccccc}
V_{1}^{\prime} & 0 & 0 & P F_{1} & P F_{1}+C^{T} F_{2} & P \\
* & W_{2} & 0 & 0 & 0 & 0 \\
* & * & W_{3} & 0 & 0 & 0 \\
* & * & * & -\varepsilon_{2} I & 0 & 0 \\
* & * & * & * & V_{2}^{\prime} & -F_{2}^{T} L^{T} \\
* & * & * & * & * & -I \\
* & * & * & * & * & * \\
* & * & * & * & * & * \\
* & * & * & * & * & * \\
P D+C^{T} D_{w} & P & P-C^{T} L^{T} \\
0 & & 0 & 0 & \\
0 & & 0 & 0 & \\
0 & & 0 & 0 \\
0 & & 0 & 0 \\
0 & & 0 & 0 \\
V_{3}^{\prime} & & -D_{w}^{T} L^{T} & 0 \\
* & & -I & 0 \\
* & & * & -I
\end{array}\right]<0
$$

where

$$
\begin{aligned}
M= & 2 Q Q_{0}+2 Q_{0} Q-2 Q_{0} Q_{0}+C^{T} L^{T} L_{0} C \\
& +C^{T} L_{0}^{T} L C-C^{T} L_{0}^{T} L_{0} C+A^{T} Q+Q A+C^{T} C \\
N^{\prime}= & F_{f}^{T} L^{T} L_{0} F_{f}+F_{f}^{T} L_{0}^{T} L F_{f}-F_{f}^{T} L_{0}^{T} L_{0} F_{f} \\
& -\gamma_{2}^{2} I+F_{f}^{T} F_{f}
\end{aligned}
$$

$$
\begin{aligned}
V_{1}^{\prime}= & A^{T} P+P A+C^{T} C-3 P P_{0}-3 P_{0} P+3 P_{0} P_{0} \\
& -C^{T} L^{T} L_{0} C-C^{T} L_{0}^{T} L C+C^{T} L_{0}^{T} L_{0} C \\
V_{2}^{\prime}= & -\varepsilon_{3} I-F_{2}^{T} L^{T} L_{0} F_{2}-F_{2}^{T} L_{0}^{T} L F_{2}+F_{2}^{T} L_{0}^{T} L_{0} F_{2} \\
V_{3}^{\prime}= & -\gamma_{1}^{2} I+D_{w}^{T} D_{w}+\varepsilon_{1} D_{w}^{T} F_{2} F_{2}^{T} D_{w}-D_{w}^{T} L^{T} L_{0} D_{w} \\
& -D_{w}^{T} L_{0}^{T} L D_{w}+D_{w}^{T} L_{0}^{T} L_{0} D_{w} \\
W_{2}= & {\left[\begin{array}{cc}
-\gamma_{1}^{2} I+\left\|F_{2}\right\| H_{1}^{T} H_{1}+\varepsilon_{3} H_{1}^{T} H_{1}^{T} & H_{1}^{T} \\
H_{1} & -\varepsilon_{1} I
\end{array}\right] } \\
W_{3}= & -\gamma_{1}^{2} I+\varepsilon_{2} H_{2}^{T} H_{2}
\end{aligned}
$$

Remark 3 When $P=P_{0}, Q=Q_{0}$ and $L=L_{0}$, it is obvious that Theorem 2 and Theorem 3 are equivalent.

Based on Theorem 3, it is possible to construct an iterative LMI algorithm similar to that in Algorithm 1. However, such algorithm will not be proposed here because of space limit.

\section{Finite Frequency Case}

In the previous sections, $\left\|H_{r f}(s)\right\|_{-}^{[0,+\infty)}$ is considered over the full frequency spectrum, i.e. $\bar{\omega}=\infty$ in (6). However, in real applications, it would be preferred to consider the fault sensitivity within a finite low frequency range including $\mathrm{DC}(\omega=0)$, i.e. $\bar{\omega}$ is a finite number. Moreover, $\left\|H_{r f}(s)\right\|_{-}^{[0,+\infty)}$ is always zero when $F_{f}$ is not of full column rank. In this case, the result proposed in Section 3 is no longer applicable. Unfortunately, such full column rank constraint on $F_{f}$ is often not satisfied in practice. Therefore, it is necessary to consider the case of $\left\|H_{r f}(s)\right\|_{\text {_ }}$ over finite frequency range in which case the constraint on $F_{f}$ can be avoided. In this section, $F_{f}$ is assumed to be not of full column rank.

In summary, for the finite frequency case, condition (9) in Problem 1 will be replaced by

$$
\left\|H_{r f}(s)\right\|_{-}^{[0, \bar{\omega}]}>\gamma_{2} \quad \text { for } 0 \leq \bar{\omega}<\infty
$$

At first, we have the following lemma which is a variant of [15, Lemma 4].

Lemma 4 Given transfer functions $W(s)$ and $M(s)$ such that

$$
\sup _{\omega \in[0, \bar{\omega}]} \bar{\sigma}[W(j \omega)]=\delta \quad, \quad\|W(s)+M(s)\|_{-}>\alpha
$$

then

$$
\|M(s)\|_{-}^{[0, \bar{\omega}]}>\alpha-\delta
$$

Suppose the state space realization of $W(s)$ is given by $\left(A_{r}, B_{r}, C_{r}, D_{r}\right)$. A realization of $T_{r f}(s)+W(s)$ is

$$
\begin{aligned}
(\tilde{A}, \tilde{B}, \tilde{C}, \tilde{D})= & \left(\left[\begin{array}{cc}
A_{r} & 0 \\
0 & A-L C
\end{array}\right],\right. \\
& {\left.\left[\begin{array}{c}
B_{r} \\
F-L F_{f}
\end{array}\right],\left[\begin{array}{ll}
C_{r} & C
\end{array}\right], D_{r}+F_{f}\right) }
\end{aligned}
$$

Thus, based on Lemma 2 and Lemma 4, we obtain the following theorem with proof omitted. 
Theorem 4 Consider system (5) and a given stable state space realization $\left(A_{r}, B_{r}, C_{r}, D_{r}\right)$ of $W(s)$ with $F_{f}+$ $D_{r}$ being of full column rank. Let $\delta:=\sup _{\omega \in[0, \bar{\omega}]} \bar{\sigma}[W(j \omega)]$, and $\gamma_{1}>0, \gamma_{2}>0$. Then system (5) is asymptotically stable and satisfies (8) and

$$
\left\|H_{r f}(s)\right\|_{-}^{[0, \bar{\omega}]}>\gamma_{2}
$$

if there exist a matrix $P>0$, symmetric matrices $Q_{1}$, $Q_{3}$, and matrices $Q_{2}$, and $L$ such that MIs (13) and (18) hold.

$$
\left[\begin{array}{cc}
A_{r}^{T} Q_{1}+Q_{1} A_{r} & A_{r}^{T} Q_{2}+Q_{2}(A-L C) \\
+C_{r}^{T} C_{r} & +C_{r}^{T} C \\
* & (A-L C)^{T} Q_{3}+Q_{3}(A-L C) \\
* & +C^{T} C \\
& * \\
Q_{1} B_{r}+Q_{2} F-Q_{2} L F_{f} \\
+C_{r}^{T} D_{r}+C_{r}^{T} F_{f} \\
Q_{2}^{T} B_{r}+Q_{3} F-Q_{3} L F_{f} \\
+C^{T} D_{r}+C^{T} F_{f} \\
-\left(\gamma_{2}+\delta\right)^{2} I+D_{r}^{T} D_{r}+D_{r}^{T} F_{f} \\
+F_{f}^{T} D_{r}+F_{f}^{T} F_{f}
\end{array}\right]>0
$$

Remark 4 For Theorem 4, it is possible to obtain an algorithm similar to that in Algorithm 1. The solution may be different depending on various chosen $W(s)$. It is obvious that $W(s)$ plays an important role in Theorem 4. Generally speaking, a better $W(s)$ should be chosen to satisfy: (i) $\delta=\sup _{\omega \in[0, \bar{\omega}]} \bar{\sigma}[W(j \omega)]$ is small; (ii) $\inf _{\omega \in\left[\omega_{2},+\infty\right)} \underline{\sigma}[W(j \omega)]$ is large with $\omega_{2}>\bar{\omega}$.

\section{Numerical Simulation}

In this section, a numerical simulation is given to demonstrate the applicability of the proposed approaches. Consider the uncertain linear system (1) with parameters as follows:

$$
\begin{aligned}
& A=\left[\begin{array}{cccc}
-5.2 & 0.65 & 6.5 & 1.3 \\
-1.56 & -2.6 & 0 & 2.6 \\
-1.3 & 0 & -1.3 & 0 \\
-0.26 & 0 & 3.9 & -1.95
\end{array}\right] \text {, } \\
& B .=F=\left[\begin{array}{c}
1 \\
1 \\
2 \\
-2
\end{array}\right], \quad D=\left[\begin{array}{cc}
1 & 0.06 \\
-3 & 0.04 \\
2 & -0.08 \\
1 & 0.02
\end{array}\right] \text {, } \\
& C=\left[\begin{array}{cccc}
-0.3 & 0.3 & 0 & 0.3 \\
0.3 & 0 & 0.3 & 0
\end{array}\right] \text {, } \\
& D_{\omega}=\left[\begin{array}{cc}
0.15 & 0.012 \\
0.3 & 0.015
\end{array}\right], \quad F_{f}=\left[\begin{array}{l}
0 \\
0
\end{array}\right] \text {, } \\
& F_{1}=\left[\begin{array}{c}
0.3 \\
0 \\
0 \\
0.3
\end{array}\right], \quad F_{2}=\left[\begin{array}{c}
0.25 \\
0.25
\end{array}\right], \\
& H_{1}=\left[\begin{array}{llll}
0.2 & 0 & 0 & 0.2
\end{array}\right], \quad H_{2}=0.375
\end{aligned}
$$

It is obvious that $\left\|H_{r f}(s)\right\|_{-}^{[0,+\infty)}$ is always zero since
$F_{f}$ is not of full column rank. Thus, Problem 1 will be investigated over a finite frequency range $[0,0.1]$ instead of the whole frequency range. With the aid of the techniques proposed in Section 4, a frequency weighting transfer matrix $W(s)$ is selected as

$$
W(s)=\left[\begin{array}{c}
\frac{50(s+0.1)}{s+100} \\
0
\end{array}\right]=\left[\begin{array}{c}
50-\frac{70.6753^{2}}{s+100} \\
0
\end{array}\right]
$$

then a state space realization $\left(A_{r}, B_{r}, C_{r}, D_{r}\right)$ of $W(s)$ is given by

$$
\begin{array}{ll}
A_{r}=\left[\begin{array}{cc}
-100 & 0 \\
0 & -100
\end{array}\right], \quad B_{r}=\left[\begin{array}{c}
70.6753 \\
0
\end{array}\right], \\
C_{r}=\left[\begin{array}{cc}
-70.6753 & 0 \\
0 & 0
\end{array}\right], \quad D_{r}=\left[\begin{array}{c}
50 \\
0
\end{array}\right]
\end{array}
$$

Note that $F_{f}+D_{r}$ is of full column rank and

$$
\delta=\sup _{\omega \in[0,0.1]} \bar{\sigma}[W(j \omega)]=0.0707 .
$$

Based on Algorithm 1, observer gain is designed by using MATLAB 6.1 with the LMI toolbox. A curve illustrating the variation of the value of $\gamma=\frac{\gamma_{1}}{\gamma_{2}}$ in the iteration is shown in Figure 1. After 60 times of iterations, $\gamma$ approaches 0.62 with $\gamma_{1}=0.36$ and $\gamma_{2}=0.58$, and the corresponding observer gain $\mathcal{L}$ is

$$
\mathcal{L}=\left[\begin{array}{cc}
-0.1263 & 3.6037 \\
2.2069 & -10.4713 \\
-4.9770 & 9.2223 \\
-3.6970 & 5.5797
\end{array}\right]
$$

The goal of Algorithm 1 is to design an observer gain such that the disturbance is attenuated and $\left\|H_{r_{f}}(s)\right\|_{-}^{[0,0.1]}$ is made large simultaneously. The results between case 1 (observer gain is the zero matrix) and case 2 (observer gain is $\mathcal{L}$ from Algorithm RFDODP1) are compared to illustrate the effectiveness of optimization. Attention is focused on the improvement in $\left\|H_{r f}(s)\right\|_{-0,0.1]}^{[0,0.1}$. The numerical comparison shows that $\left\|H_{r f}(s)\right\|_{-}^{[0,0.1]}$ is increased by $110.7 \%$ (from 0.5535 to 1.1662 ).

The system is simulated with unit step input $u(t)$ and unknown input $w(t)=\left[\sin (2 t) e^{-0.1 t} \quad \cos (2 t) e^{-0.1 t}\right]^{T}$. For an actuator fault $f(t)$ such that $f(t)=-0.7 ; t \geq 10 \mathrm{~s}$ and $f(t)=0$ elsewhere, Figure 2 shows the evolution of residual evaluation function $r(t)$ responses due to observer gain $\mathcal{L}$. From Figure 2, we can see that, despite the influence of uncertainties and unknown inputs, a threshold at \pm 0.4 can easily be imposed on the residual signals to indicate the occurrence of fault at the time $t=10.48 \mathrm{~s}$, i.e. the fault $f(t)$ can be detected $0.48 \mathrm{sec}$ after its occurrence.

\section{Conclusion}

In this paper, the RFD problem for linear time-invariant systems under structured uncertainties is investigated with the aid of the $H_{\infty}$ norm and $H_{-}$index optimization techniques and the LMI approach. It aims at enhancing the robustness to disturbance without sacrificing the FD 
sensitivity. First, RFD problem is handled for system without structured uncertainties. A necessary and süfficient condition is proposed for the design of RFD observer. Then result for uncertain system is given. This has not been systematically treated in previous fault detection studies. Moreover, the RFD problem over finite frequency range is also investigated, in which case the condition on the rank of $D$ for a system $(A, B, C, D)$ is no longer required. Numerical simulation is used to illustrate the effectiveness of the results.

\section{References}

[1] S. X. Ding, T. Jeinsch, P. M. Frank, and E. L. Ding. A unified approach to the optimization of fault detection systems. International Journal of Adaptive Control and Signal Processing, 14(7):725-745, 2000.

[2] X. Ding and P. M. Frank. Fault detection via optimally robust detection filters. In Proc. 28th IEEE Conference on Decision and Control, volume 2, pages 1767-1772, 1989.

[3] X. Ding, L. Guo, and T. Jeinsch. A characterization of parity space and its application to robust fault detection. IEEE Transactions on Automatic Control, 44(2):337-343, 1999.

[4] P. M. Frank. Fault diagnosis in dynamic systems using analytical and knowledge-based redundancy: a survey and some new results. Automatica, 26(3):459474, 1990.

[5] P. M. Frank and X. Ding. Survey of robust residual generation and evaluation methods in observerbased fault detection systems. J. Proc. Control, 7(6):403$424,1997$.

[6] J. Gertler. Survey of model-based failure detection and isolation in complex plants. IEEE Control Systems Magazine, 8(6):3-11, 1988.

[7] M. Hou and R. J. Patton. An LMI approach to $H_{-} / H_{\infty}$ fault detection observers. In Proc. of the UKACC int. Conf. on Contr.: CONTROL'96, IEE, pages 305-310, Univ. of Exter, UK, 1996.

[8] R. Isermann. Process fault detection based on modelling and estimation methods: A survey. Automatica, 20(4):387-404, 1984.

[9] X. $\mathrm{Li}$ and C. E. de Souza. Delay-dependent robust stability and stabilization of uncertain linear delay systems: a linear matrix inequality approach. IEEE Transactions on Automatic Control, 42:1144-1148, 1997.

[10] J. Liu, J. L. Wang, and G. H. Yang. An LMI approach to $H_{-} / H_{\infty}$ fault detection observers. to be presented at ACC'03, Denver, Colorado, USA, June 2003.

[11] J. Liu, J. L. Wang, and G. H. Yang. Worst-case fault detection observer design: An LMI approach. In The 2002 International Conference on Control and Automation, pages 1243-1247, Xiamen, China, 2002.

[12] R. J. Patton. Robust model-based fault diagnosis: the state of the art. In IFAC Symp. Fault Detection,
Supervision and Safety for Technical Process, pages 1-27, Finland, 1994. Academic Press.

[13] R. J. Patton, P. M. Frank, and R. N. Clark. Fault Diagnosis in Dynamic Systems: Theory and Applications. Prentice Hall, London, 1989.

[14] R. J. Patton and M. Hou. $H_{\infty}$ estimation and robust fault detection. In Proc. of the 1997 European Control Conference, Brussels, Belgium, 1997.

[15] M. L. Rank and H. Niemann. Norm based design of fault detectors. International Journal of Control, 72(9):773-783, 1999.

[16] H. B. Wang, J. Lam, S. Ding, and M. Zhong. Iterative LMI algorithms for fault detection with unknown inputs. International Journal of Control, 2001. Revised and re-submitted.

[17] A. S. Willsky. A survey of design methods for failure detection in dynamic systems. Automatica, 12(4):601$611,1976$.

[18] S. Xu, J. Lam, and C. Yang. $H_{\infty}$ and positive real control for linear neutral delay systems. IEEE Transactions on Automatic Control, 46(8):1321-1326, 2001.

[19] M. Zhong, S. X. Ding, J. Lam, and H. B. Wang. An LMI approach to design robust fault detection filter for uncertain LTI systems. Automatica, 39:543-550, 2003.

[20] K. Zhou, J. C. Doyle, and K. Glover. Robust and Optimal Control. Prentice Hall, New Jersey, 1996.

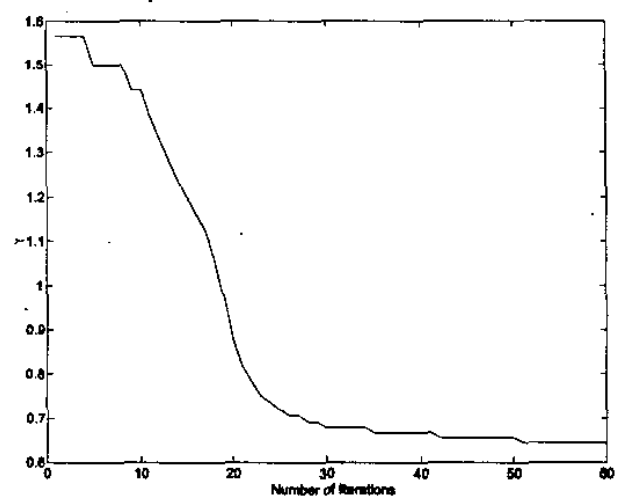

Figure 1. Variation of performance index $\gamma=\frac{\gamma_{1}}{\gamma_{2}}$

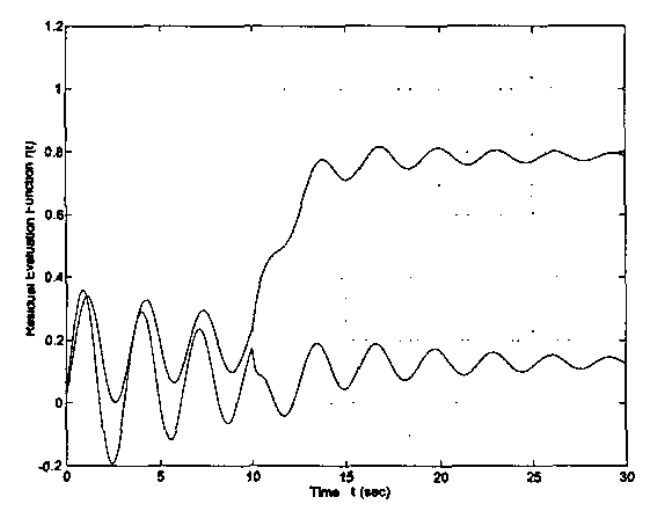

Figure 2. Evolution of residual evaluation function 